\title{
Getting a Kick out of the Stellar Disk(s) in the Galactic Center
}

\author{
H. B. Perets, G. Kupi and T. Alexander
}

Weizmann Institute of Science, POB 26, Rehovot 76100, Israel.

\begin{abstract}
Recent observations of the Galactic center revealed a nuclear disk of young OB stars, in addition to many similar outlying stars with higher eccentricities and/or high inclinations relative to the disk (some of them possibly belonging to a second disk). Binaries in such nuclear disks, if they exist in non-negligible fractions, could have a major role in the evolution of the disks through binary heating of this stellar system. We suggest that interactions with/in binaries may explain some (or all) of the observed outlying young stars in the Galactic center. Such stars could have been formed in a disk, and later on kicked out from it through binary related interactions, similar to ejection of high velocity runaway OB stars in young clusters throughout the galaxy.
\end{abstract}

Keywords. Galaxy: center, black hole physics, stars: kinematics, binaries: general

Recent observations have revealed the existence of many young OB stars in the galactic center (GC). Accurate measurements of the orbital parameters of these stars give strong evidence for the existence of a massive black hole $(\mathrm{MBH})$ which govern the dynamics in the GC (Eisenhauer et al. 2005). Most of the young stars are observed to be OB stars in the central $0.5 \mathrm{pc}$ around the MBH. Many of them are observed in a coherent stellar disk or two perpendicular disks configurations (Lu et al. 2006; Paumard et al. 2006). Others are observed to be have inclined and/or eccentric $(>0.5)$ orbits relative to the stellar disks (hereafter outliers). The inner $0.04 \mathrm{pc}$ near the $\mathrm{MBH}$ contain only young B-stars, that possibly have a different origin (e.g. Levin 2007; Perets, Hopman \& Alexander 2007).

It was suggested that the disk stars have been formed a few Myrs years ago in a fragmenting gaseous disk (Nayakshin \& Cuadra 2005; Levin 2007). However, the origin of outliers from the disk is difficult to explain in this way. These stars are observed to have very similar stellar properties to the young disk stars (types, lifetimes), but have more inclined and/or eccentric orbits. Many suggestions have been made for the origin of these stars (Milosavljević \& Loeb 2004; Paumard et al. 2006; Alexander, Begelman \& Armitage 2007; Yu, Lu \& Lin 2007, and references therein). Here we suggest a different process in which young stars in the GC stellar disks were kicked into high inclinations and/or eccentricities, in a similar way to OB runaway stars ejected from open clusters. Such a scenario could explain some of the puzzling orbital properties of the young stars in the GC.

A considerable fraction of the early OB stars in the solar neighborhood have large peculiar velocities $\left(40 \leqslant v_{p e c} \leqslant 200 \mathrm{~km} \mathrm{~s}^{-1}\right.$; e.g. Hoogerwerf, de Bruijne \& de Zeeuw 2001) and are observed in isolated locations; these are the so-called runaway stars (Blaauw 1961). Two mechanisms are thought to eject OB runaway stars, both involve binarity (or higher multiplicity). In the binary supernova scenario (BSS; Blaauw 1961) a star is kicked at high velocity following a supernova explosion of its companion. In the dynamical ejection scenario (DES; Poveda, Ruiz \& Allen 1967) runaway stars are ejected through gravitational interactions between stars in dense, compact clusters. The DES is more likely for the disk stars, given the short lifetime of the disk.

The binary properties of stars in the GC are unknown, but observations of eclipsing binaries in the GC suggests they are not fundamentally different from that observed in 

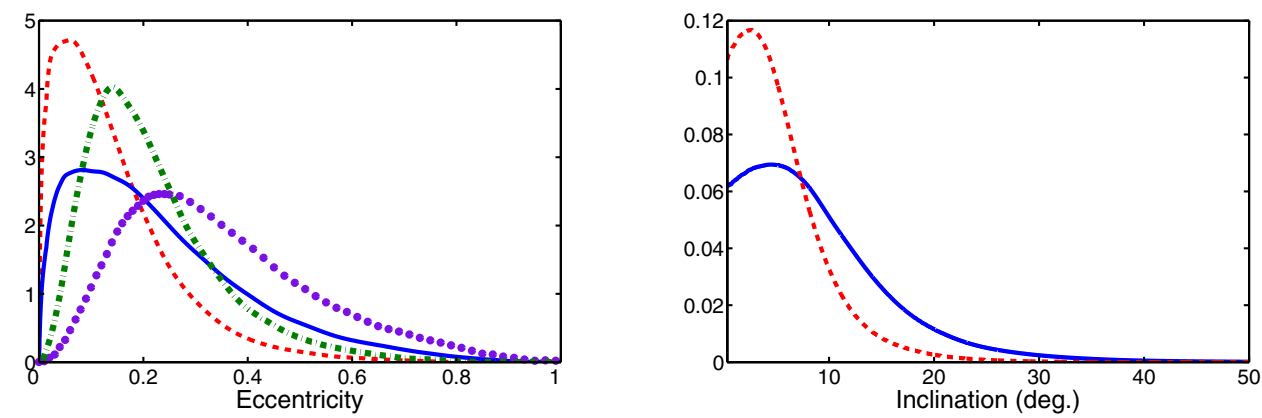

Figure 1. Left: Eccentricity distribution of outliers in the Galactic center. Monte-Carlo results assuming different models for the Maxwellian kick velocity distributions; isotropic, $v \sim 100 \mathrm{~km}$ $\mathrm{s}^{-1}$ (solid), isotropic, $v \sim 60 \mathrm{~km} \mathrm{~s}^{-1}$ (dashed), planar (co-planar with the disk), $v \sim 100 \mathrm{~km}$ $\mathrm{s}^{-1}$ (dotted) and planar, $v \sim 60 \mathrm{~km} \mathrm{~s}^{-1}$ (dash-dotted). Right: Inclination distribution of outliers in the Galactic center. Monte-Carlo results assuming different models for the Maxwellian kick velocity distributions; isotropic, $v \sim 100 \mathrm{~km} \mathrm{~s}^{-1}$ (solid), isotropic, $v \sim 60 \mathrm{~km} \mathrm{~s}^{-1}$ (dashed).

the solar neighborhood (DePoyet et al. 2004; Martins et al. 2006; Rafelski et al. 2007). The conditions in the stellar disks in the GC are highly favorable for the DES given the high stellar densities $\left(>10^{6} \mathrm{pc}^{-3}\right)$, the low velocity dispersion and the masses of the stars in the stellar disk. It is thus quite likely that dynamical ejection in the disk is more frequent and efficient than in normal Galactic star forming regions. In order to escape the GC cusp, a disk star initially bounded to the $\mathrm{MBH}$ needs a kick velocity of a few $\times 10^{2} \mathrm{~km} \mathrm{~s}^{-1}$. Most of the observed OB runaways in the galaxy do not reach such high velocities. Therefore runaways from the stellar disk would not escape, and would remain in the cusp. However, such kicks could considerably change the orbits of these stars. In Fig. 1 we show the inclination and eccentricity distribution of OB stars that were formed in a disk on circular orbits and were kicked out of the disk with velocities typical of OB runaways $\left(40-200 \mathrm{~km} \mathrm{~s}^{-1}\right)$. Stars in the disk with near circular Keplerian orbits, would be kicked into more eccentric and inclined orbits (on average). Their orbits should also display some correlations between inclination, eccentricity and mass. Such "failed" runaways could possibly explain the outliers from and inside the disks in the $\mathrm{GC}$, which are difficult to explain otherwise.

\section{References}

Alexander, R. D., Begelman, M. C., \& Armitage, P. J. 2007, ApJ 654, 907

Blaauw, A. 1961, Bull. Astro. Inst. Netherlands 15, 505, 265

Depoy, D. L. et al. 2004, ApJ 617, 1127

Eisenhauer, F. et al. 2005, ApJ 628, 246

Hoogerwerf, R., de Bruijne, J. H. J., \& de Zeeuw, P. T. 2001, A\&A 365, 49

Levin, Y. 2007, MNRAS 374, 515

Lu, J. R. et al. 2006, J. Phys.: Conf. Ser. 54, 279

Martins, F. et al. 2006, ApJL 649, L103

Milosavljević, M. \& Loeb, A. 2004, ApJL 604, L45

Nayakshin, S. \& Cuadra, J. 2005, A\&A 437, 437

Paumard, T. et al. 2006, ApJ 643, 1011

Perets, H. B., Hopman, C., \& Alexander, T. 2007, ApJ 656, 709

Poveda, A., Ruiz, J., \& Allen, C. 1967, Bol. Obs. Tonantzintla y Tacubaya 4, 86

Rafelski, M. et al. 2007, ApJ 659, 1241

Yu, Q., Lu, Y., \& Lin, D. N. C. 2007, ApJ 666, 919 